\title{
Optimization of Albumin Secretion and Metabolic Activity of Cytochrome P450 1A1 of Human Hepatoblastoma HepG2 Cells in Multicellular Spheroids by Controlling Spheroid Size
}

\author{
Tomoko Nishikawa, ${ }^{a}$ Yutaro Tanaka, ${ }^{a}$ Makiya Nishikawa, ${ }^{*, a, b}$ Yuka Ogino, ${ }^{a}$ Kosuke Kusamori, ${ }^{a}$ \\ Narumi Mizuno, ${ }^{a}$ Yuya Mizukami, ${ }^{a}$ Kazunori Shimizu, ${ }^{b, c, d}$ Satoshi Konishi, ${ }^{b, d, e}$ \\ Yuki Takahashi, ${ }^{a}$ and Yoshinobu Takakura ${ }^{a, b}$ \\ ${ }^{a}$ Department of Biopharmaceutics and Drug Metabolism, Graduate School of Pharmaceutical Sciences, Kyoto \\ University; Sakyo-ku, Kyoto 606-8501, Japan: ${ }^{b}$ Institute for Innovative NanoBio Drug Discovery and Development \\ Graduate School of Pharmaceutical Sciences, Kyoto University; Sakyo-ku, Kyoto 606-8501, Japan: ${ }^{c}$ Department of \\ Biotechnology, Graduate School of Engineering, Nagoya University; Furo-cho, Chikusa-ku, Nagoya, Aichi 464-8603, \\ Japan: ${ }^{d}$ Department of Mechanical Engineering, Ritsumeikan University; Kusatsu, Shiga 525-8577, Japan: and \\ ${ }^{e}$ Ritsumeikan-Global Innovation Research Organization, Ritsumeikan University; Kusatsu, Shiga 525-8577, Japan. \\ Received October 25, 2016; accepted December 20, 2016
}

Multicellular spheroids are useful as three-dimensional cell culture systems and for cell-based therapies. Their successful application requires an understanding of the consequences of spheroid size for cellular functions. In the present study, we prepared multicellular spheroids of different sizes using the human hepatoblastoma HepG2 cells, as hepatocytes are frequently used for in vitro drug screening and cell-based therapy. Precise polydimethylsiloxane-based microwells with widths of $360,450,560$, and $770 \mu \mathrm{m}$ were fabricated using a micromolding technique. Incubation of $\mathrm{HepG} 2$ cells in cell culture plates containing the microwells resulted in the formation of HepG2 spheroids with average diameters of 195, 320, 493, and $548 \mu \mathrm{m}$. The cell number per spheroid positively correlated with its diameter, and the viability of HepG2 cells was 94\% or above for all samples. The smallest HepG2 spheroids showed the highest albumin secretion. On the other hand, the metabolic activity of 7-ethoxyresorufin, a fluorometric substrate for CYP1A1, increased with increasing spheroid size. These results indicate that controlling spheroid size is important when preparing HepG2 spheroids and that the size of HepG2 spheroids greatly influences the cellular function of HepG2 cells in the spheroids.

Key words albumin; CYP1A1; human hepatoblastoma HepG2 cell; size control; spheroid

Three-dimensional multicellular spheroids possess greater in vivo tissue-like morphology and function than two-dimensional monolayered cells. Therefore, multicellular spheroids are considered useful in vitro systems for drug screening, including tests of drug metabolism and toxicity, ${ }^{1-6)}$ and for studying tumor metastasis. ${ }^{7,8)}$ Furthermore, such spheroids have attracted much attention for application in cell-based therapy ${ }^{9-11)}$ and as a building block for the construction of large tissues. ${ }^{12,13)}$ In a previous study, we demonstrated that spheroid formation greatly prolonged the survival of insulinsecreting NIT-1 cells after transplantation in mice.9)

Spheroid size is a key parameter to determine the functions and properties of spheroids. It is reported that oxygen concentration is decreased in the core of the spheroids of human hepatoblastoma HepG2 cells, hepatocytes, and tumor cells. $^{14-17)}$ This affects the expression of various molecules, including hypoxia inducible factor (HIF)-1. In addition, paracellular or transcellular transport is required in order for the inner cells to take up extracellular materials and to release secretory products into the culture media. Despite their importance, details regarding the relationship between spheroid size and cellular functions remain to be elucidated. In particular, few studies have investigated the effect of spheroid sizes over $300 \mu \mathrm{m}$ on their functions and properties.

Investigating the effects of spheroid size requires the preparation of size-controlled, multicellular spheroids. Several techniques for spheroid formation have been reported, including the spinner flask, hanging drop, and liquid overlay methods. ${ }^{18-20)}$ However, a common problem with these techniques is the difficulty in controlling spheroid size. In contrast, microfabrication is suitable for the development of sizecontrolled microwells with narrow size distribution. ${ }^{10,21,22)}$ In a previous study, we demonstrated that polydimethylsiloxane (PDMS)-based microwells constructed using a microfabrication technique could be used to obtain spheroids with a very narrow size distribution. ${ }^{23)}$ We also found that coating the microwells with poly( $N$-isopropylacrylamide) (PNIPAAm), a thermoresponsive polymer, increased the quality of the spheroids, since the coating prevented the cells from adhering to the PDMS-based microwells. ${ }^{23)}$

In the present study, we developed PDMS-based microwells of different sizes in order to prepare variably sized multicellular spheroids. HepG2 cells were used for the study as they represent one of the most extensively used cell lines to evaluate drug metabolism and toxicity. ${ }^{3,24,25)}$ Production of various proteins is an index of liver function. Albumin is the most abundant serum protein produced by hepatocytes. ${ }^{26}$ ) Another important liver function is the metabolism of various compounds, including drugs. CYP enzymes are the major enzymes involved in drug metabolism. CYP1A1 is one of the major CYP enzymes that is involved in the metabolic activation of aromatic hydrocarbons and is a major source of variability in drug pharmacokinetics, ${ }^{27)}$ and its expression in HepG2 cells was reported in many studies. Therefore, albumin 
and CYP1A1 were selected as indicators of cellular function.

\section{MATERIALS AND METHODS}

Chemicals Dulbecco's modified Eagle's medium (DMEM, low glucose type) was obtained from Nissui Pharmaceutical Co., Ltd. (Tokyo, Japan). Fetal bovine serum (FBS) was obtained from Equitech-Bio, Inc. (Kerrville, TX, U.S.A.). A PDMS prepolymer and the curing agent (Silpot 184) were purchased from Toray-Dow Corning Co. (Tokyo, Japan). PNIPAAm was obtained from Polysciences Inc. (Warrington, PA, U.S.A.). Trypan blue stain solution $(0.5 \%)$ was obtained from Nacalai Tesque Inc. (Kyoto, Japan). Trypsin was obtained from Becton Dickinson and Co. (Mansfield, MA, U.S.A.). Ethylenediaminetetraacetic acid, disodium salt, dihydrate (EDTA-2Na) was obtained from Dojindo Laboratories (Kumamoto, Japan). All other chemicals were of the highest grade commercially available.

Cells and Cell Culture HepG2 cells were purchased from American Type Culture Collection (Manassas, VA, U.S.A.) and grown in DMEM supplemented with 10\% heat-inactivated $\mathrm{FBS}, 0.15 \% \mathrm{NaHCO}_{3}, 100 \mathrm{U} / \mathrm{mL}$ potassium penicillin $\mathrm{G}$, and $100 \mathrm{mg} / \mathrm{mL}$ streptomycin sulfate at $37^{\circ} \mathrm{C}$ in humidified air containing $5 \% \mathrm{CO}_{2}$.

Preparation of PDMS-Based Microwells Four types of micropillar array with different widths, i.e., 360, 450, 560, and $770 \mu \mathrm{m}$, were fabricated as previously described. The microwells were named small, medium, large, and extra large according to their size.

PNIPAAm Coating of PDMS-Based Microwells The PDMS-based microwell sheet, within a 6-well culture plate, was coated with PNIPAAm as previously described. ${ }^{23)}$

Preparation of HepG2 Spheroids HepG2 cells $\left(5 \times 10^{6}\right.$ cells for small, medium, and large microwells, or $1 \times 10^{7}$ cells for extra large microwells) suspended in DMEM were added to PNIPAAm-coated PDMS-based microwells, placed in 6-well culture plates, and incubated for $72 \mathrm{~h}$ at $37^{\circ} \mathrm{C}$ in a humidified $5 \% \mathrm{CO}_{2}$ incubator. The culture plates were shaken at a speed of $65 \mathrm{rpm}$ for the first $3 \mathrm{~h}$. Large and extra large microwells were transferred from the 6 -well culture plates to a $10 \mathrm{~cm}$ dish after $3 \mathrm{~h}$ of incubation to increase the volume of culture media and to maintain high cell viability. Subsequently, these microwells were shaken at a speed of $40 \mathrm{rpm}$ for an additional $69 \mathrm{~h}$ to enable uniform concentration of oxygen and nutrients in culture media. The culture medium was changed at 3,24 , and $48 \mathrm{~h}$ of incubation. To maintain the PNIPAAm coating, the coated microwells were kept at $37^{\circ} \mathrm{C}$ during the experiments. After $72 \mathrm{~h}$ incubation, the culture plates were exposed to room temperature, which caused the PNIPAAm coating to dissolve. Then, HepG2 spheroids were detached from the PDMS-based microwells and washed twice with phosphate buffered saline (PBS) to remove any remaining PNIPAAm. Thereafter, HepG2 spheroids were selected using micropipettes. Each HepG2 spheroid was named as small, medium, large, or extra large according to the PDMSbased microwells used for their preparation. HepG2 spheroids were observed under a Biozero microscope and the diameter of the spheroids was measured using the BZ analyzer software. The number and viability of HepG2 cells in one spheroid was measured by counting the cells stained with trypan blue solution after dispersion of the spheroid using a trypsin-EDTA solution $(0.25 \%$ trypsin, $0.02 \%$ EDTA). To the 20 spheroids in
$20 \mu \mathrm{L}$ of PBS was added $80 \mu \mathrm{L}$ of the trypsin-EDTA solution. After 5 min incubation at $37^{\circ} \mathrm{C}$, the mixture was pipetted to disintegrate the spheroids into dispersed HepG2 cells.

Albumin Secretion from HepG2 Cells HepG2 spheroids containing approximately $1 \times 10^{5}$ HepG2 cells were added to each well of a 6 -well culture plate. To achieve this, 67, 25, 12, and 7 spheroids were used for small, medium, large, and extra large HepG2 spheroids, respectively. Suspended (dispersed) HepG2 cells were prepared by detaching cells from a culture dish using a trypsin-EDTA solution ( $0.25 \%$ trypsin, $0.02 \%$ EDTA) and added to a well at the same number $\left(1 \times 10^{5}\right.$ cells/ well). After $24 \mathrm{~h}$ incubation, the culture supernatants were collected and stored at $-80^{\circ} \mathrm{C}$ until use. The concentration of human albumin in the supernatants was determined using the Human Albumin enzyme-linked immunosorbent assay (ELISA) Quantitation Set (Bethyl Laboratories Inc., Montgomery, TX, U.S.A.), according to the manufacturer's protocol.

Ethoxyresorufin- $\boldsymbol{O}$-deethylase Assay To measure CYP1A1 activity, ethoxyresorufin- $O$-deethylase (EROD) assay was performed as previously reported. ${ }^{28)}$ In brief, suspended HepG2 cells and HepG2 spheroids approximately corresponding with $8 \times 10^{4}$ HepG2 cells were added to a well in the 24-well plate with $10 \mu \mathrm{M}$ of 7-ethoxyresorufin. A number of HepG2 spheroids used in the EROD assay is 35, 20, 14, and 9 spheroids of small, medium, large, and extra large HepG2 spheroids, respectively. After $1 \mathrm{~h}$ incubation under lightproof condition, the fluorescence of metabolites from 7-ethoxyresorufin by HepG2 cells and spheroids was detected by FluoroMax-4 spectrofluorometer (HORIBA, Ltd., Kyoto, Japan).

Statistical Analysis Differences were statistically evaluated using one-way ANOVA followed by Tukey's test for multiple comparisons. A value of $p<0.05$ was considered statistically significant.
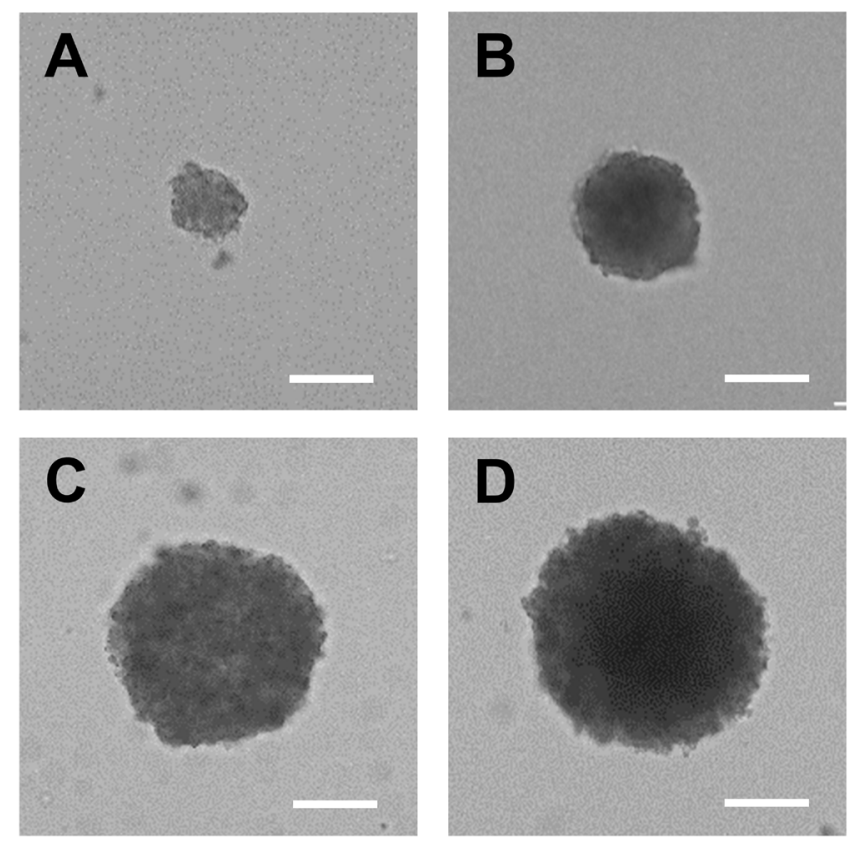

Fig. 1. Microscopic Images of HepG2 Spheroids

Images of HepG2 spheroids were taken under a microscope, and typical images are shown: (A) small, (B) medium, (C) large, and (D) extra large HepG2 spheroids. Scale bar in each image represents $200 \mu \mathrm{m}$. 
A

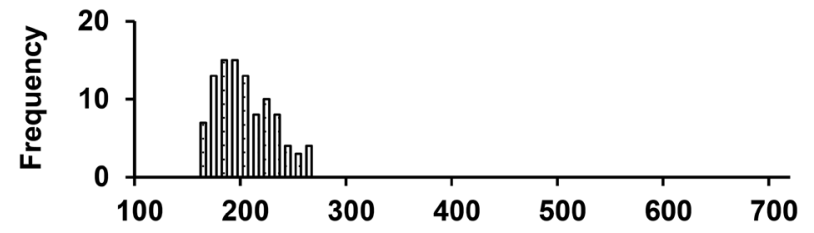

B

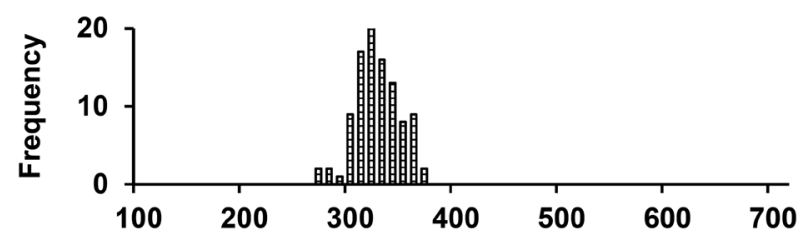

C

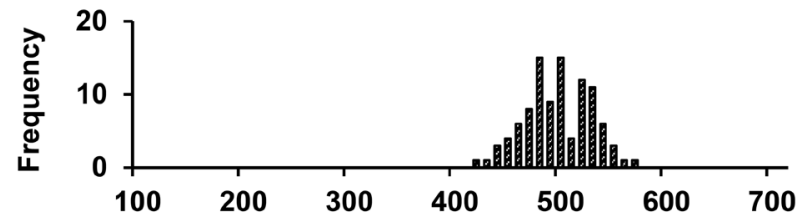

D

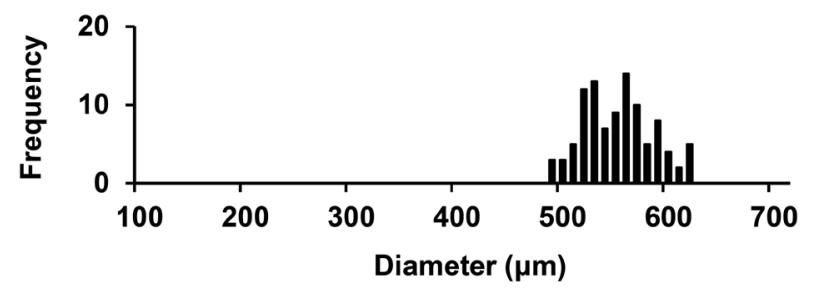

Fig. 2. Size Distributions of HepG2 Spheroids

The histograms of the diameter of 100 spheroids were measured under a microscope: (A) small, (B) medium, (C) large, and (D) extra large HepG2 spheroids.

A

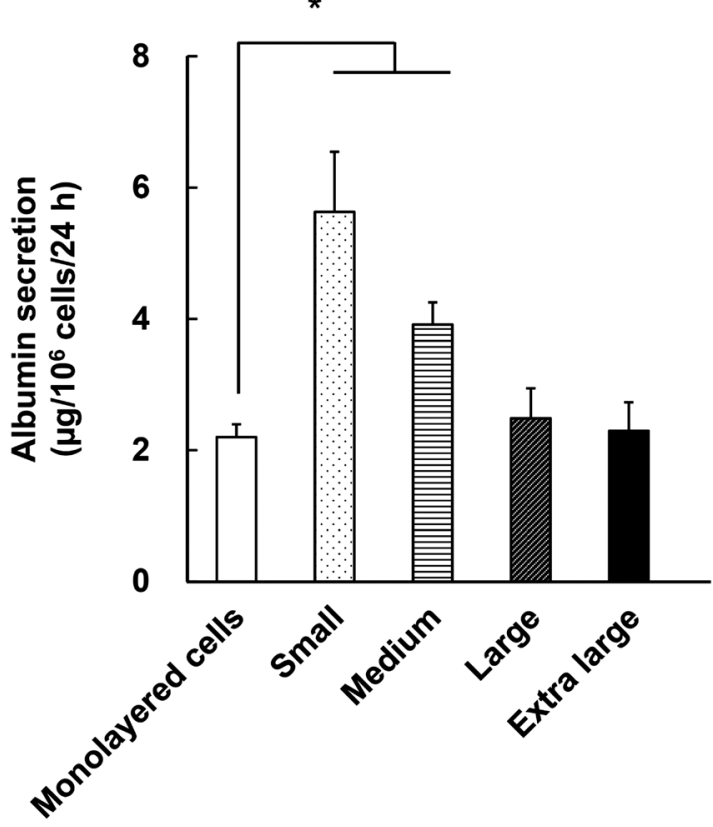

\section{RESULTS}

Size of HepG2 Spheroids and Viability of HepG2 Cells in the Spheroids Figures 1A-D show microscopic images of the small, medium, large, and extra large HepG2 spheroids, and Figs. 2A-D show histograms of their diameter. All HepG2 spheroids had narrow size distribution. The average diameter of the small, medium, large, and extra large HepG2 spheroids was 195, 320, 493, and $548 \mu \mathrm{m}$, respectively (Table 1). The number of HepG2 cells per spheroid was approximately $1500,4000,8500$, and 13000 for the small, medium, large, and extra large HepG2 spheroids, respectively (Table 1). Cell viability was $94 \%$ or greater for all spheroids. Cell viability of monolayered HepG2 cells treated with the trypsin-EDTA solution was $98.5 \%$ or more (data not shown), suggesting that the treatment of HepG2 spheroids with the trypsin-EDTA solution hardly affected the viability of the cells and spheroid formation slightly reduced it.

Secretion of Albumin from Monolayered HepG2 Cells and HepG2 Spheroids We measured the concentration of albumin in the culture medium of monolayered HepG2

Table 1. Characteristics of HepG2 Spheroids

\begin{tabular}{lccc}
\hline \hline & Diameter $(\mu \mathrm{m})$ & Cell number & Viability (\%) \\
\hline Small & $195 \pm 27$ & $1500 \pm 220$ & $95.4 \pm 2.9$ \\
Medium & $320 \pm 21$ & $4080 \pm 360$ & $97.6 \pm 1.7$ \\
Large & $493 \pm 32$ & $8450 \pm 510$ & $96.5 \pm 1.7$ \\
Extra large & $548 \pm 33$ & $13000 \pm 1300$ & $94.1 \pm 1.1$ \\
\hline
\end{tabular}

Diameter is expressed as the mean \pm S.D. of 100 measurements. Cell number and viability are expressed as the mean \pm S.D. of five measurements.
B

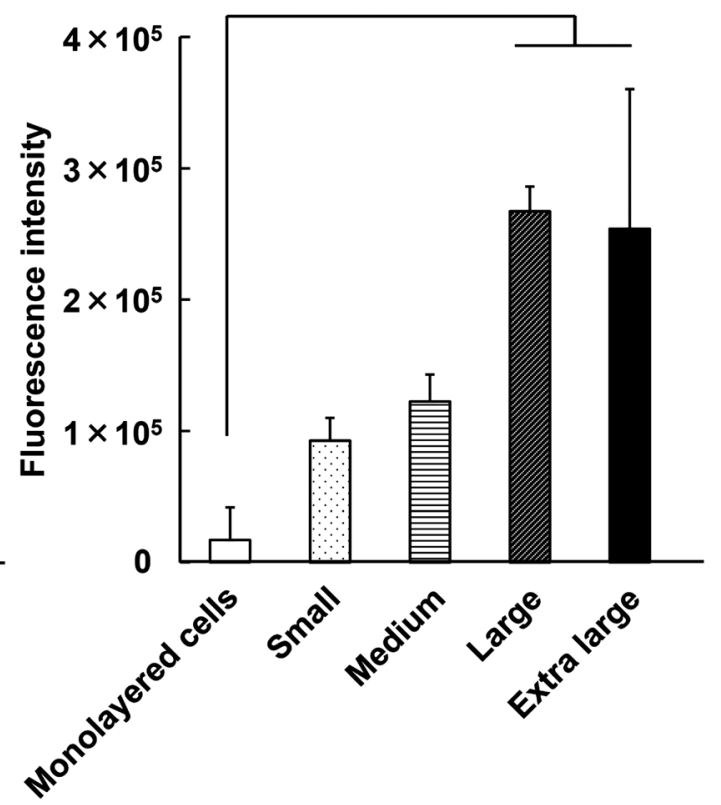

Fig. 3. Cellular Functions of Monolayered HepG2 Cells and HepG2 Spheroids: Albumin Secretion (A) and EROD Activity (B) of HepG2 Cells

(A) Monolayered HepG2 cells (suspended before use) or HepG2 spheroids were added to each well of a 6 -well culture plate at approximately $1 \times 10^{5}$ cells/well. The concentration of albumin in culture media was measured $24 \mathrm{~h}$ after incubation. Results are expressed as the mean \pm S.D. of five measurements. A representative experiment of three independent experiments with similar results is presented. (B) $8 \times 10^{4}$ monolayerd HepG2 cells and HepG2 spheroids which correspond with $8 \times 10^{4}$ HepG2 cells were incubated with $10 \mu \mathrm{M}$ of 7-ethoxyresorufin for $1 \mathrm{~h}$. After the incubation, the supernatants were used for measurement of fluorescence intensity with FluoroMax-4. Results are expressed as the mean \pm S.D. of three measurements. A representative experiment of five independent experiments with similar results is presented. $* p<0.05$ versus the monolayer group. 
cells and HepG2 spheroids (Fig. 3A). The small and medium HepG2 spheroids released significantly higher amounts of albumin than the monolayered HepG2 cells, and large and extra large HepG2 spheroids.

EROD Assay of Monolayered HepG2 Cells and HepG2 Spheroids The activity of isozyme CYP1A1 was measured by the EROD assay (Fig. 3B). The activity elevated with increasing spheroid size. The activity of the large and extra large HepG2 spheroids was significantly higher than that of monolayered HepG2 cells.

\section{DISCUSSION}

The method based on PDMS-based microwells developed in the previous study is superior to other techniques in terms of productivity and uniformity in size. ${ }^{23)}$ The present study clearly demonstrates that PDMS-based microwells can effectively prepare different sized multicellular spheroids, with a narrow size distribution (Figs. 1, 2, Table 1).

Previous reports have discussed the relationship between spheroid size and cellular functions. For instance, it was shown that albumin secretion from HepG2 spheroids with diameters between 125 and $250 \mu \mathrm{m}$ increased as spheroid size increased. ${ }^{24)}$ On the other hand, levels of secretion of $\alpha$-fetoprotein, albumin, and fibrinogen in HepG2 spheroids, with diameters ranging between 100 and $250 \mu \mathrm{m}$, was independent of spheroid size. ${ }^{25)}$ However, little is known about the properties of HepG2 spheroids larger than $250 \mu \mathrm{m}$.

It was reported that large HepG2 spheroids with diameters of $180 \mu \mathrm{m}$ or greater contain dead cells in their central regions because of minimal or no transport of oxygen and nutrients. ${ }^{24,29)}$ These reports are inconsistent with our results, which demonstrate the high viability of large and extra large HepG2 spheroids ( $94 \%$ or above). The increased volume of culture media added to large and extra large microwells could explain this. Supporting this hypothesis, when large and extra large HepG2 spheroids were incubated for $72 \mathrm{~h}$ in 6-well culture plates, as were the small and medium HepG2 spheroids, the average diameter of the large and extra large HepG2 spheroids was smaller (361 and $517 \mu \mathrm{m}$, respectively) than those used for this experiment (493 and $548 \mu \mathrm{m}$; Table 1). Furthermore, the viability of large and extra large HepG2 spheroids was lower (74.3 and $65.9 \%$, respectively) than those used (97 and 94\%). In addition, it has been reported that increasing the glucose concentration of the culture media reduces the number of dead cells in the spheroids of EMT6/Ro mouse mammary tumor cells and MGH-U1 human bladder cancer cells. ${ }^{17,30)}$ Therefore, it can be speculated that the viability of large and extra large HepG2 spheroids was high in this experiment because the cells in the spheroids were supplied with sufficient nutrients during the culture period.

Previous studies have shown that the cellular functions of HepG2 cells and hepatocytes are improved by spheroid formation because of cell-cell interactions. ${ }^{1,2,23,31)}$ The extensive cell-cell interaction could explain the enhanced albumin secretion and EROD activity in HepG2 cells when formulated in multicellular spheroids.

Protein synthesis and metabolism are two major functions of hepatocytes. In the present study, albumin secretion and CYP1A1 activity were selected as indicators to evaluate the function of HepG2 spheroids, because they can be measured with high sensitivity. Numerous reports have indicated that a low concentration of amino acids directly reduces the rate of albumin synthesis both in intact animals and in isolated hepatocytes. ${ }^{32,33)}$ Similarly, the rate of albumin synthesis of rats fed with protein free or low-protein diets for 2-3 weeks was lower than that of rats fed with normal diets; moreover, decreased albumin secretion was also observed in isolated rat hepatocytes. ${ }^{34-37)}$ On the other hand, previous studies reported that the formation of spheroids using human fatal hepatocytes and human hepatocellular carcinoma cells, including HepG2 cells, resulted in higher expression of CYP1A1 proteins and genes, respectively, compared to the monolayered cells. ${ }^{38,39)}$ Moreover, it has been reported that the exposure of male SpragueDawley (SD) rats to hypoxic conditions for $48 \mathrm{~h}$ increased the EROD activity in the liver. ${ }^{40)}$ As the size of HepG2 spheroids became larger, hypoxic areas in HepG2 spheroids will increase, because the core region of multicellular spheroids is known to be under hypoxic conditions. Consequently, it is likely that the elevation in the EROD activity in the present study is attributed to the spheroid size-dependent expansion of hypoxic areas in the spheroids.

In conclusion, we successfully developed HepG2 spheroids with precisely controlled size using precise PDMS-based microwells with widths of $360,450,560$, and $770 \mu \mathrm{m}$. We found that the spheroid formation generally improved the function of HepG2 cells, but the spheroid size differently affected the albumin secretion and CYP1A1 activity of HepG2 cells. These results indicate that controlling spheroid size is important and that the size of HepG2 spheroids greatly influences the cellular function of HepG2 cells in the HepG2 spheroids.

Acknowledgments This work was supported in part by a Grant-in-Aid for Exploratory Research (No. 23659283) and a Grant-in-Aid for Scientific Research (S) (No. 26220206) from Japan Society for the Promotion of Science (JSPS), by a Grant from the Ritsumeikan Global Innovation Research Organization (R-GIRO), and by an Institute for Integrated Cell-Material Sciences (iCeMS) Cross-Disciplinary Research Promotion Project of Kyoto University.

Conflict of Interest The authors declare no conflict of interest.

\section{REFERENCES}

1) Takayama K, Kawabata K, Nagamoto $Y$, Kishimoto $K$, Tashiro $K$, Sakurai F, Tachibana M, Kanda K, Hayakawa T, Furue MK, Mizuguchi H. 3D spheroid culture of hESC/hiPSC-derived hepatocytelike cells for drug toxicity testing. Biomaterials, 34, 1781-1789 (2013).

2) Sakai Y, Tanaka T, Fukuda J, Nakazawa K. Alkoxyresorufin $O$ dealkylase assay using a rat hepatocyte spheroid microarray. $J$. Biosci. Bioeng., 109, 395-399 (2010).

3) Torisawa YS, Takagi A, Nashimoto Y, Yasukawa T, Shiku H, Matsue T. A multicellular spheroid array to realize spheroid formation, culture, and viability assay on a chip. Biomaterials, 28, 559-566 (2007)

4) Vinci M, Gowan S, Boxall F, Patterson L, Zimmermann M, Court W, Lomas C, Mendiola M, Hardisson D, Eccles SA. Advances in establishment and analysis of three-dimensional tumor spheroidbased functional assays for target validation and drug evaluation. BMC Biol., 10, 29 (2012). 
5) Godugu C, Patel AR, Desai U, Andey T, Sams A, Singh M. AlgiMatrix $^{\mathrm{TM}}$ based $3 \mathrm{D}$ cell culture system as an in-vitro tumor model for anticancer studies. PLoS ONE, 8, e53708 (2013).

6) Lee DW, Choi Y, Seo YJ, Lee M, Jeon SY, Ku B, Kim S, Yi SH, Nam D. High-throughput screening (HTS) of anticancer drug efficacy on a micropillar/microwell chip platform. Anal. Chem., 86, 535-542 (2014).

7) Krohn A, Song Y, Muehlberg F, Droll L, Beckmann C, Alt E. CXCR4 receptor positive spheroid forming cells are responsible for tumor invasion in vitro. Cancer Lett., 280, 65-71 (2009).

8) Okochi M, Takano S, Isaji $Y$, Senga $T$, Hamaguchi $M$, Honda $H$. Three-dimensional cell culture array using magnetic force-based cell patterning for analysis of invasive capacity of BALB $/ 3 \mathrm{~T} 3 / \mathrm{v}$-src. Lab. Chip, 9, 3378-3384 (2009).

9) Kusamori K, Nishikawa M, Mizuno N, Nishikawa T, Masuzawa A, Shimizu K, Konishi S, Takahashi Y, Takakura Y. Transplantation of insulin-secreting multicellular spheroids for the treatment of type 1 diabetes in mice. J. Control. Release, 173, 119-124 (2014).

10) Jun Y, Kang AR, Lee JS, Jeong GS, Ju J, Lee DY, Lee S. 3D coculturing model of primary pancreatic islets and hepatocytes in hybrid spheroid to overcome pancreatic cell shortage. Biomaterials, 34, 3784-3794 (2013).

11) Bhang SH, Cho S, La W, Lee T, Yang HS, Sun A, Baek S, Rhie $\mathrm{J}$, Kim B. Angiogenesis in ischemic tissue produced by spheroid grafting of human adipose-derived stromal cells. Biomaterials, 32, 2734-2747 (2011).

12) Mironov V, Visconti RP, Kasyanov V, Forgacs G, Drake CJ, Markwald RR. Organ printing: tissue spheroids as building blocks. Biomaterials, 30, 2164-2174 (2009)

13) Jakab K, Norotte C, Damon B, Marga F, Neagu A, Besch-Williford CL, Kachurin A, Church KH, Park H, Mironov V, Markwald R, Vunjak-Novakovic G, Forgacs G. Tissue engineering by self-assembly of cells printed into topologically defined structures. Tissue Eng. Part A, 14, 413-421 (2008).

14) Anada T, Fukuda J, Sai Y, Suzuki O. An oxygen-permeable spheroid culture system for the prevention of central hypoxia and necrosis of spheroids. Biomaterials, 33, 8430-8441 (2012).

15) Glicklis R, Merchuk JC, Cohen S. Modeling mass transfer in hepatocyte spheroids via cell viability, spheroid size, and hepatocellular functions. Biotechnol. Bioeng., 86, 672-680 (2004).

16) Curcio E, Salerno S, Barbieri G, De Bartolo L, Drioli E, Bader A. Mass transfer and metabolic reactions in hepatocyte spheroids cultured in rotating wall gas-permeable membrane system. Biomaterials, 28, 5487-5497 (2007).

17) Mueller-Klieser W, Freyer JP, Sutherland RM. Influence of glucose and oxygen supply conditions on the oxygenation of multicellular spheroids. Br. J. Cancer, 53, 345-353 (1986).

18) Okubo H, Matsushita M, Kamachi H, Kawai T, Takahashi M, Fujimoto T, Nishikawa K, Todo S. A novel method for faster formation of rat liver cell spheroids. Artif. Organs, 26, 497-505 (2002).

19) Tung YC, Hsiao AY, Allen SG, Torisawa Y, Ho M, Takayama S. High-throughput 3D spheroid culture and drug testing using a 384 hanging drop array. Analyst, 136, 473-478 (2011).

20) Metzger W, Sossong D, Bächle A, Pütz N, Wennemuth G, Pohlemann $\mathrm{T}$, Oberringer $\mathrm{M}$. The liquid overlay technique is the key to formation of co-culture spheroids consisting of primary osteoblasts, fibroblasts and endothelial cells. Cytotherapy, 13, 1000-1012 (2011).

21) Sakai Y, Yoshida S, Yoshiura Y, Mori R, Tamura T, Yahiro K, Mori H, Kanemura Y, Yamasaki M, Nakazawa K. Effect of microwell chip structure on cell microsphere production of various animal cells. J. Biosci. Bioeng., 110, 223-229 (2010).

22) Bernard AB, Lin C, Anseth KS. A microwell cell culture platform for the aggregation of pancreatic $\beta$-cells. Tissue Eng. Part C Methods, 18, 583-592 (2012).

23) Shimizu K, Kusamori K, Nishikawa M, Mizuno N, Nishikawa T, Masuzawa A, Katano S, Takahashi Y, Takakura Y, Konishi S. Poly-
( $N$-isopropylacrylamide)-coated microwell arrays for construction and recovery of multicellular spheroids. J. Biosci. Bioeng., 115, 695-699 (2013).

24) Tamura T, Sakai Y, Nakazawa K. Two-dimensional microarray of HepG2 spheroids using collagen/polyethylene glycol micropatterned chip. J. Mater. Sci. Mater. Med., 19, 2071-2077 (2008).

25) Sakai Y, Nakazawa K. Technique for the control of spheroid diameter using microfabricated chips. Acta Biomater., 3, 1033-1040 (2007).

26) Jeschke MG. The hepatic response to thermal injury: is the liver important for postburn outcomes? Mol. Med., 15, 337-351 (2009).

27) Vakharia DD, Liu N, Pause R, Fasco M, Bessette E, Zhang QY, Kaminsky LS. Polycyclic aromatic hydrocarbon/metal mixtures: effect on PAH induction of CYP1A1 in human HEPG2 cells. Drug Metab. Dispos., 29, 999-1006 (2001).

28) Wilkening S, Stahl F, Bader A. Comparison of primary human hepatocytes and hepatoma cell line Hepg2 with regard to their biotransformation properties. Drug Metab. Dispos., 31, 1035-1042 (2003).

29) Fukuda J, Okamura K, Nakazawa K, Ijima H, Yamashita $Y$, Shimada M, Shirabe K, Tsujita E, Sugimachi K, Funatsu K. Efficacy of a polyurethane foam/spheroid artificial liver by using human hepatoblastoma cell line (Hep G2). Cell Transplant., 12, 51-58 (2003).

30) Tannock IF, Kopelyan I. Influence of glucose concentration on growth and formation of necrosis in spheroids derived from a human bladder cancer cell line. Cancer Res., 46, 3105-3110 (1986).

31) Chang TT, Hughes-Fulford M. Monolayer and spheroid culture of human liver hepatocellular carcinoma cell line cells demonstrate distinct global gene expression patterns and functional phenotypes. Tissue Eng. Part A, 15, 559-567 (2009).

32) Kirsch RE, Saunders SJ, Frith L, Wicht S, Kelman L, Brock JF. Plasma amino acid concentration and the regulation of albumin synthesis. Am. J. Clin. Nutr., 22, 1559-1562 (1969).

33) John DW, Miller LL. Regulation of net biosynthesis of serum albumin and acute phase plasma proteins. Induction of enhanced net synthesis of fibrinogen, alpha1-acid glycoprotein, alpha2 (acute phase)-globulin, and haptoglobin by amino acids and hormones during perfusion of the isolated normal rat liver. J. Biol. Chem., 244, 6134-6142 (1969).

34) Morgan EH, Peters T Jr. The biosynthesis of rat serum albumin. V. Effect of protein depletion and refeeding on albumin and transferrin synthesis. J. Biol. Chem., 246, 3500-3507 (1971).

35) Grant AG, Hoffenberg R. The effect of dietary protein deprivation on protein synthesis in the isolated liver parenchymal cell. $B r . J$. Nutr., 38, 255-260 (1977).

36) Hoffenberg R, Gordon A, Black E. Albumin synthesis by the perfused rat liver. A comparison of methods with special reference to the effect of dietary protein deprivation. Biochem. J., 122, 129-134 (1971)

37) Smith JE, Lunn PG. Albumin-synthesizing capacity of hepatocytes isolated from rats fed diets differing in protein and energy content. Ann. Nutr. Metab., 28, 281-287 (1984)

38) Matsushita T, Nakano K, Nishikura Y, Higuchi K, Kiyota A, Ueoka R. Spheroid formation and functional restoration of human fetal hepatocytes on poly-amino acid-coated dishes after serial proliferation. Cytotechnology, 42, 57-66 (2003).

39) Terashima J, Goto S, Hattori H, Hoshi S, Ushirokawa M, Kudo K, Habano W, Ozawa S. CYP1A1 and CYP1A2 expression levels are differentially regulated in three-dimensional spheroids of liver cancer cells compared to two-dimensional monolayer cultures. Drug Metab. Pharmacokinet., 30, 434-440 (2015).

40) Okamoto T, Mitsuhashi M, Fujita I, Sindhu RK, Kikkawa Y. Induction of cytochrome P450 1A1 and 1A2 by hyperoxia. Biochem. Biophys. Res. Commun., 197, 878-885 (1993). 